\title{
Zoonotic leishmaniasis and heartworm disease in Italy: changing epidemiological scenarios and future perspectives
}

\section{Jairo Mendoza-Roldan}

Universita degli Studi di Bari Aldo Moro

Giovanni Benelli

Universita degli Studi di Pisa

\section{Rossella Panarese}

Universita degli Studi di Bari Aldo Moro

\section{Roberta latta}

Universita degli Studi di Bari Aldo Moro

\section{Tommaso Furlanello}

San Marco Veterinary clinic

\section{Frederic Beugnet}

Boehringer Ingelheim Animal Health

\section{Andrea Zatelli}

Universita degli Studi di Bari Aldo Moro

Domenico Otranto ( $\sim$ domenico.otranto@uniba.it )

Dipartimento di Medicina Veterinaria, Università degli studi di Bari https://orcid.org/0000-0002-7518476X

\section{Research}

Keywords: Aedes mosquitoes, canine vector-borne diseases, Dirofilaria immitis, Leishmania infantum, sandflies

Posted Date: February 24th, 2020

DOl: https://doi.org/10.21203/rs.2.24377/v1

License: (c) (i) This work is licensed under a Creative Commons Attribution 4.0 International License.

Read Full License 


\section{Abstract}

Background: For long time, canine leishmaniosis (CanL) was considered endemic in the southern, central, and insular regions of Italy, whereas heartworm disease (HW) by Dirofilaria immitis in the northern region and in the swampy Po valley. Following the reports of new foci of both diseases, in this study we update the distribution patterns and occurrence of new foci of CanL and HW discussing the main drivers for the changes in the epidemiology of these two important zoonotic canine vector-borne diseases.

Methods: Based on the statistical analyses of serological assays $(n=90,633)$ on CanL and HW performed by reference diagnostic centres in Italy over a ten-year period (2009-2019), the distribution patterns of both diseases were herein presented along with the occurrence of new foci.

Results: Results highlighted the changing distribution patterns of CanL $v s$ HW disease in Italy. CanL is now also endemic in the northern regions and HW has endemic foci in central and southern regions and islands. Significant differences in CanL and HW prevalence among the study macroareas were detected. The overall prevalence of CanL was $28.2 \%$ in Southern Italy and Islands, $29.6 \%$ in Central Italy and $21.6 \%$ in Northern Italy and that of HW of $2.83 \%$ in northern Italy, $7.75 \%$ in central Italy and $4.97 \%$ in southern Italy and islands. HW prevalence significantly varied over years $\left(\chi^{2}=108.401, d . f .=10, p<0.0001\right)$, gradually increasing from $0.77 \%$ in 2009 to values ranging from $5.19-8.47 \%$ in $2016-2017$.

Conclusions: The new epidemiological scenarios have been discussed according to a range of factors (e.g. environmental modifications, occurrence of competent insect vectors, transportation of infected animals to non-endemic areas, chemoprophylaxis or vector preventative measures), which may affect the current distribution. Overall, results advocate for epidemiological surveillance programs, more focussed preventative and control measures even in areas where few or none cases of both diseases have been diagnosed.

\section{Background}

In the last decades, canine vector-borne diseases (CVBDs) have been expanding worldwide due to several factors linked with increase in pet travelling along with owners, relocation of stray animals from endemic to previously non-endemic regions, as well as to the modification of the ecology of arthropod vectors and, importantly, environmental modifications [1-3]. In some cases, this resulted in the spreading of new parasites and pathogens, and related infections, in previously non-endemic geographical areas, which poses major concerns to veterinary practitioners and, in the case of zoonotic ones, to public health officials [2]. Protozoan Leishmania infantum Nicolle and nematode Dirofilaria immitis Leidy represent paradigmatic examples of the modification in the distribution of the CVBDs they cause (i.e., canine leishmaniosis, CanL, and heartworm disease, HW). The expansion of the above infections has been related to the distribution of their vectors (i.e., for CanL, sandflies of the genus Phlebotomus in the Mediterranean region; for HW, several mosquito species, belonging to the genera Aedes, Anopheles and Culex, [4-7]). 
In specific geographical contexts, such as Italy, where both CVBDs have been endemic for long time [8], their ecology and distribution have been studied and new foci were reported by a retrospective analysis focusing on the period from 1990 to 2009 [1]. Indeed, until 1990, CanL was considered endemic in the southern, central, and insular regions of Italy, whereas dirofilariosis in the northern region and in the Po valley $[9,10]$. Sporadic case reports suggested that the distribution of both CVBDs has been changing, in that, dirofilariosis expanded towards the southern regions and CanL to the northern regions [1]. This phenomenon could have been the effect of chemoprophylaxis measures by using macrocyclic lactones $(\mathrm{ML})$ in endemic areas of northern Italy and of the absence of such preventative measures in remaining central and southern regions [11]. Indeed, the only species of filarioids historically diagnosed in southern Italy have been Acanthocheilonema reconditum and Dirofilaria repens, causing less pathogenic subcutaneous filariosis [12,13].

Meanwhile, the perception of clinicians and parasitologists has most likely changed, resulting into an increase of the request for diagnostic tests of $D$. immitis infection in central-southern Italy, as well of CanL in northern regions [8]. In this scenario, the aim of the present study is to present results of serological assays performed by reference diagnostic centres in Italy over a ten-year period (20092019), therefore updating the distribution patterns and occurrence of new foci of CanL and HW.

\section{Methods}

\section{Databases of serological tests in dogs from Italy}

Databases from two diagnostic reference centres (hereafter reported as database A and B) were analysed (total number of serum samples $=90,633$ ). Italian macroareas were defined as northern, central and southern/islands macroareas (Fig. 1).

The complied data was standardized to observe significant variations on positive serological results and records of new non-endemic foci. The database A included 62,928 records of dogs collected in a 10-year period (2009-2019) throughout the Italian peninsula, including information regarding breed, sex, age. Different serological (Novatec ${ }^{\circledR}$ kit for CanL and Dirocheck Zoetis ${ }^{\circledR}$ for HW) Enzyme Linked Immune Sorbent Assay (ELISA) tests were used. Of the total number, 78 records were excluded due to uncertainty of the origin of the samples.

The second dataset (B) included information of 26,258 of dogs examined from northern Italy (i.e., Emilia Romagna, Lombardia, Trentino-Alto Adige, and Piedmont), central Italy (i.e., Lazio and Tuscany), and mainly from southern Italy and islands (i.e., Basilicata, Calabria, Campania, Apulia, Sicily and Sardinia) in a five-year period (2015 - 2019). This dataset also comprised information regarding breed, sex, age, and serological (VetLine ${ }^{\circledR}$ Leishmania for CanL and Filarcheck $96 \AA$ for HW) ELISA, and an indirect semiquantitative immunofluorescence test (MegaFLUO LEISH® for leishmaniosis) with positive or negative results. Of the total number, 70 records were excluded due to uncertainty of the origin of the samples, and other 423 samples from the northern and central regions were not considered for the statistical analysis 
given that the data was biased by transportation of positive animal from endemic areas. Both datasets included results for CanL and HW.

\section{Statistical analyses}

Differences in the prevalence of CanL and HW infection in the studied dogs from northern, central and southern Italy over time were analysed by JMP 9 (SAS) by using weighted generalized linear models (GLZ) with a binomial distribution to test model positive and negative serological outcomes. For each parasitosis, a GLZ with two fixed factors was used to assess significant differences in CanL or HW prevalence among the study macroareas over years: $y=X \beta+\varepsilon$ where $y$ is the vector of the observation [i.e. serological outcome: positive $=1$, negative $=0, X$ is the incidence matrix, $B$ is the vector of fixed effects (the study macroarea (northern, central or southern Italy and islands) and years)] and $\varepsilon$ is the vector of the random residual effects $(p=0.05)$.

Then, a dataset was created for each study macroarea and a GLZ with two fixed factors was used to evaluate significant differences in CanL or HW prevalence among the region and years within a given macroarea; the structure of the GLZ was identical to the above described one, with two fixed effects [i.e. the study region and year]. A $p$-value of 0.05 was used as threshold to assess significant differences among values. To verify that the changing distribution patterns of CanL and HW were not random, a contingency analysis assessing the relationship between the CanL and HW prevalence in the various macroareas, regions and study years was also carried out [14].

Serological data were presented in terms of bi-annual and cumulative prevalence; distribution maps for CanL and HW were generated using QGIS version 3.4.4-Madeira, to compare new non-endemic localities with those reported previously [15].

\section{Results}

Overall, the number of serological tests for CanL $(n=80,309)$ performed in the three areas of Italy is reported in Table 1, being higher in southern Italy (37.7\% of all tests performed) than in central Italy (31\%), and northern Italy (24.7\%). Conversely, the overall number of tests requested for the diagnosis of HW throughout Italy $(n=10,324)$ was significantly lower $(11.3 \%$ of all the requested serological tests) than for CanL, with a relative high number in northern Italy ( $51.7 \%$ of all tests performed) and the lowest in southern Italy (Table 1 ).

Results showed a significant difference in CanL prevalence among the different study macroareas $\left(\chi^{2}=218.564\right.$, d.f. $\left.=2, p<0.0001\right)$, with an overall prevalence of $28.2 \%$ in southern Italy and islands, $29.68 \%$ in central Italy and $21.62 \%$ in northern Italy (Table $1 ;$ Fig. 2 ). The impact of the study year on CanL prevalence in Italy was also significant $\left(\chi^{2}=559.846\right.$, d.f. $\left.=10, p<0.0001\right)$, with values $>30 \%$ in 2011,2012 , 2014 and 2015. HW prevalence showed significant differences among the study macroareas $\left(\chi^{2}=114.879\right.$, d.f. $\left.=2, p<0.0001\right)$, being of $2.83 \%$ in northern Italy, $7.75 \%$ in central Italy and $4.97 \%$ in southern Italy and islands. On the whole Italian territory, HW prevalence significantly varied over years 
$\left(\chi^{2}=108.401\right.$, d.f. $\left.=10, p<0.0001\right)$, gradually increasing from $0.77 \%$ in 2009 to values ranging from 5.19$8.47 \%$ in 2016-2017 (Table 1; Fig. 3).

A detailed analysis over CanL and HW prevalence trend over time in the three Italian macroareas was provided by assessing the impact of the study year and region on CanL and HW prevalence. In northern Italy, CanL prevalence showed significant differences over the study years $\left(\chi^{2}=286.277\right.$, d.f. $=10$, $p<0.0001$ ), being highest in 2012 and 2014 (i.e., 33.51\% and 27.27\%, respectively) (Fig. 2). Notably, significant variations in CanL prevalence among the various northern Italy regions were recorded $\left(\chi^{2}=190.657\right.$, d.f. $\left.=7, p<0.0001\right)$, with the highest values in Piedmont, Trentino Alto Adige, Valle d'Aosta, and Friuli Venezia Giulia $(28.93 \%, 27.59 \%, 27.40 \%$ and $27.17 \%$, respectively). The study year and region significantly impacted HW prevalence in northern Italy $\left(\chi^{2}=56.954\right.$, d.f. $=10, p<0.0001 ; \chi^{2}=40.555$, d.f. $=7$, $p<0.0001$, respectively), being highest prevalence rate observed in 2016 (7.20\%) (Fig. 3). Highest prevalence values were retrieved in Val d'Aosta (11.36\%), Trentino Alto Adige (7.41\%) and Piedmont (6.29\%), while the lowest regional prevalence was in Veneto $(2.12 \%)$.

In central Italy, both the study year and region led to significant differences in CanL prevalence $\left(\chi^{2}=371.252\right.$, d.f. $=10, p<0.0001 ; \chi^{2}=609.769$, d.f. $=5, p<0.0001$, respectively $)$. It was higher than $30 \%$ in 2012, 2014, 2015 and 2016 (Fig. 2), with the highest values in Lazio (38.52\%), followed by Umbria (35.61\%) and Abruzzo (34.10\%). HW prevalence was also affected by the study year $\left(\chi^{2}=55.333\right.$, d.f. $=10$, $p<0.0001$ ), showing values $>10 \%$ in 2016 (i.e., 12.1\%) and 2017 (i.e, 13.32\%) (Fig. 3). Significant differences in the prevalence of HW were noted among central Italy $\left(\chi^{2}=80.975\right.$, d.f. $=4, p<0.0001$, respectively) with the highest values recorded in Tuscany (11.48\%) and Marche (7.84\%).

In southern Italy and islands, CanL prevalence was significantly different among the study years $\left(\chi^{2}=201.963\right.$, d.f. $\left.=10, p<0.0001\right)$, with values ranging from $24.55 \%$ (2017) to $50.66 \%$ (2011) (Fig. 2). CanL prevalence in this macroarea also showed significant differences among regions $\left(\chi^{2}=642.949\right.$, d.f. $=6$, $p<0.0001)$ with the highest values recorded in Molise (54.26\%), Sicily (50.18\%) and Sardinia (38.34\%). On the other hand, the study region did not play a significant role impacting HW prevalence, even if a trend was observed $\left(\chi^{2}=10.723\right.$, d.f. $=6, p=0.10$, respectively). Indeed, the largest number of analysed samples was from Apulia $(n=1608)$ followed by Basilicata $(n=80)$ and Sardinia $(n=70)$. Considering regions with sample size $>40$, the highest prevalence rates were from Sardinia and Apulia $(10 \%$, and $4.73 \%$, respectively). The effect of the study year was not significant $\left(\chi^{2}=16.145, d . f .=10, p=0.136\right)$ due to the limited number of samples examined in the years 2009-2014 (i.e., $n=117$ ), at variance with the larger samplings done in 2015-2019 (i.e., $n=1,729$ ).

Contingency results obtained analysing the separate datasets for each study macroarea are given in the Supplementary Online Material Table S1. Overall, observed changing distribution patterns of CanL and HW in Italy were not random or due to a biased sampling over the different areas and years (CanL, macroarea: $\chi^{2}=486.62$, d.f. $=2, p<0.0001$; year: $\chi^{2}=827.903$, d.f. $=10, p<0.0001$, respectively; HW, macroarea: $\chi^{2}=104.545$, d.f. $=2, p<0.0001$; year: $\chi^{2}=99.070, d . f .=10, p<0.0001$, respectively). Serological 
CanL results had a similar cumulative prevalence throughout the Italian territory (Fig. 2), showing an annual slight decrease in all the regions, from 2015 to 2018. CanL was widely distributed throughout the Italian peninsula, with a number of positive animals $>400$ in central (Lazio and Tuscany) and southern (Apulia and Basilicata) regions as well as in both Islands (Sardinia and Sicily). In addition provinces with $>300$ positive samples were in northern regions (i.e., Turin, Piedmont, and Vicenza Veneto) (Fig. 4).

The highest prevalence of HW was registered in the central regions (i.e., Tuscany and Lazio) followed by southern and northern regions with annual variation patterns throughout the observation period (Fig. 3). An overall lower number of samples was positive for $\mathrm{HW}$ compared to CanL, with $>10$ positive cases recorded in three provinces from the northern Italy (i.e., Turin, Piedmont; Genova, Liguria; and Belluno, Veneto). The province with the highest number of HW cases in central Italy (i.e., Florence, Tuscany) was surrounded by provinces with more than 20 positive samples (i.e., Bologna, Emilia Romagna; Arezzo and Prato, Tuscany), was recorded in central Italy. The largest number of positive samples in southern Italy was recorded in the Apulia region (i.e., Brindisi and Lecce provinces). No data was available for four provinces of the North (i.e., Biella, Piedmont; Lecco Lombardia; Piacenza, Emilia Romagna; and Verona, Veneto), for two provinces of southern Italy (i.e., Isernia, Molise; Vibo-Valentia, Calabria), and many provinces of the Islands (i.e., Nuoro and Oristano, Sardinia; Agrigento, Caltanissetta and Ragusa, Sicily) (Fig. 5).

\section{Discussion}

Based on the results of this large dataset $(n=90,633)$ of serological assays herein presented, the distribution patterns of CanL and HW have changed over the last ten years' period (2009-2019), also in comparison with observational data of a retrospective study, which assessed the prior decade (19992009) [1]. The seroprevalence of CanL in northern Italy increased in the examined 10 years' period (2009 to 2019$)$ from $2.1 \%$ (1999-2009; $[1,16]$ to $21.6 \%$. The results also indicate that CanL has spread progressively in the past decade in the northern Italian regions, with an overall prevalence higher than that recorded in previous studies [1,17]. For example, in the province of Bologna (Emilia Romagna) the seroprevalence increased from 6.6\% (i.e., 16 out of 245 animals examined in the period 2007-2009; [17] up to $17.16 \%$ (i.e., 217 out of 1,264 tests in this study). Overall, CanL has spread progressively in the past decades from endemic southern towards northern regions, making the whole Italian peninsula endemic for this infection. Under these circumstances, the effect of relocation of infected animals from the South to the North of Italy could not be fully assessed in the study period. Nonetheleess, the northward spread of the main sand fly vectors [i.e., Phlebotomus (Larroussius) perniciosus (Newstead) and Phlebotomus neglectus Tonnoir], which are now established in different provinces of the northern macroarea $[16,18,19]$ supports the evidence of CanL endemicity and the occurrence of new foci. In addition, clinicians' perception and awareness of the presence of CanL in non-endemic areas seem to be increased in the northern regions, with more than 21,545 tests performed (Table 1). Although the South remains the area with the largest number of tests requested, the large number of tests requested in the North and the Centre indicates that there is a clear awareness of the distribution of the disease throughout Italy. 
On the other hand, the cumulative prevalence of HW greatly increased in central (7.7\%) and southern Italy and Islands (5\%), being higher than in the North $(2.8 \%)$, which was historically considered the sole endemic area $[1,20]$. The decreased prevalence of HW in northern Italy regions could be a consequence of clinicians' awareness of the disease and thus of the continuous usage of chemoprophylactic programs in this area. Nonetheless, resistance to $\mathrm{ML}$ has been demonstrated in $D$. immitis populations from this macroarea [21], wich may represent a potential issue (not yet proven) for re-emergence of resistent strains. Moreover, the low number of tests performed for HW (10.5\% of all the requested serological tests) throughout the Italian peninsula also indicates the scant awareness of the occurrence of the disease, especially in southern regions (Table 1). Given the increased prevalence in the Centre, the South and Islands, clinicians should consider the occurrence of HW cases in non-endemic areas.

Furthermore, our analyses showed that the distribution patterns of CanL and HW in Italy are related to a significant relationship between CanL/HW prevalence and the geographical provenience (i.e., macroarea) and study years (2009-2019). Conversely, a major hindrance of this study could be that the anamnesis of animals was not available and therefore their travelling history from/to historically endemic areas for both infections (i.e., northern Italy for HW and southern and central Italy for CanL) [5,22].

However, the clustering of positive samples in some spots of CanL [e.g., the provinces of Turin (Piedmont) and Vicenza (Veneto), northern Italy; Fig. 3] and of HW [e.g., in Brindisi and Lecce (Apulia), southern Italy; Fig 4] is consistent with previous reports. Endemic foci of CanL in the same provinces of Ven.eto [23] and Piedmont [24] confirmed the above mentioned epidemiological picture with competent vectors of $L$. infantum (i.e., P. perniciosus) captured in entomological surveys performed in that area [19]. In addition, other studies reported cases of CanL and human visceral leishmaniasis diagnosed in the same area, along with their vectors, $P$. perniciosus and $P$. neglectus [25].

The southward changing pattern of HW has been detailed in previous researches [26-29]. A recent questionnaire study pointed out Brindisi and Lecce, from Apulia region, to be areas with more than 20 cases of HW caused by D. immitis [30]. The low prevalence of HW in Sicily and Sardinia could be explained by the small number of test requested or no tests performed in many provinces from these islands. Indeed, earlier studies illustrated the islands have a large number of diagnosed HW [30] as they are suitable for Dirofilaria spp. to thrive [13]. Specifically, Sardinia has the environmental, climatic and human activities (e.g., tourism with animal transportation) that could allow these nematodes to spread [5, 30]. Finally, central Italy showed a distribution pattern on which both pathogens are highly prevalent. In this macroarea, the prevalence of CanL was higher (29.6\%) of that indicated in previous surveys (up to $10 \%)[31,32]$. Hence, central Italian regions are an important pathway for the spreading of CanL from southern to northern. Notably, represent major drivers which woud deserve more investigation are the increasing in dog population and transportation of infected animals to non-endemic areas where the vectors are already present [1].

\section{Conclusions}


The large number of data strongly supports the above figure of distribution of CanL and HW. Overall, results highlighted the changing distribution patterns of CanL vs HW disease in Italy over a 10-year period (2009-2019) in which CanL is in fact now endemic in the northern Italy regions, and HW has endemic foci in the central and southern Italy and in islands. Moreover, given the number of test requested, the overall clinicians' awareness in northern Italy is increasing for CanL and diminishing for HW. The presented epidemiological picture suggests that practitioners, veterinarians and public health officials should be aware that these pathogens are distributed throughout the country, thus epidemiological surveillance, preventative and control measures should be carried out to protect dogs from CanL and HW and reduce the risk of infection in humans.

\section{Abbreviations}

CVBDs: canine vector-borne diseases; CanL: canine leishmaniosis; HW: heartworm disease; Cl: confidence interval.

\section{Declarations}

\section{Acknowledgments}

Authors thank the responsible for the two diagnostic centres for providing results of the analyses herein examined (i.e., database A - San Marco Veterinary Clinic and Laboratory, Veggiano, Padova, Italy; database B - A.C.V. Triggiano S.R.L. Laboratorio di Analisi Cliniche Veterinarie, Triggiano, Bari, Italy).

\section{Declarations}

\section{Ethics approval and consent to participate}

Not applicable.

\section{Consent for publication}

Not applicable.

\section{Availability of data and materials}

All data generated or analyzed during this study are included in this published article.

\section{Competing interests}

All authors declare that they have no competing interests.

\section{Funding}

Not applicable. 
Authors' contributions

JAMR and DO conceived the study. JAMR, GB perform the data curation, methods and anaylises of data. JAMR, GB, DO wrote the first draft of the manuscript. TF, FB, AZ, DO, RI, RP reviewed the manuscript. All authors read and approved the final manuscript.

\section{References}

1. Otranto D, Capelli G, Genchi C. Changing distribution patterns of canine vector borne diseases in Italy: leishmaniosis vs. dirofilariosis. Parasit Vectors. 2009;2 Suppl 1(Suppl 1):S2.

2. Colwell DD, Dantas-Torres F, Otranto D. Vector-borne parasitic zoonoses: emerging scenarios and new perspectives. Vet Parasitol.2011;182(1):14-21.

3. Wilke ABB, Beier JC, Benelli, G. Complexity of the relationship between global warming and urbanization - an obscure future for predicting increases in vector-borne infectious diseases. Curr Opin Insect Sci. 2019;35:1-9.

4. Killick-Kendrick R. Phlebotomine vectors of the leishmaniases: a review. Med Vet Entomol. 1990;4(1):1-24.

5. Genchi C, Mortarino M, Rinaldi L, Cringoli G, Traldi G, Genchi M. Changing climate and changing vector-borne disease distribution: the example of Dirofilaria in Europe. Vet Parasitol. 2011;176(4):295-99.

6. Morchón R, Carretón E, González Miguel J, Mellado Hernández I. Heartworm disease (Dirofilaria immitis) and their vectors in Europe-new distribution trends. Front Physiol. 2012;3:196.

7. Benelli G, Wilke ABB, Beier JC. Aedes albopictus (Asian tiger mosquito). Trends Parasitol. Forthcoming 2020.

8. Otranto D, Dantas-Torres F. Canine and feline vector-borne diseases in Italy: current situation and perspectives. Parasit Vectors. 2010;3(1):2.

9. Genchi C, Kramer LH, Rivasi F. Dirofilarial infections in Europe. Vector Borne Zoonotic Dis. 2011;11(10):1307-317.

10. Ferroglio E, Maroli M, Gastaldo S, Mignone W, Rossi L. Canine leishmaniasis, Italy. Emerg Infect Dis. 2005;11(10):1618.

11. Clemence RG, Sarasola P, Genchi C, Smith DG, Shanks DJ, Jernigan AD, et al. Efficacy of selamectin in the prevention of adult heartworm (Dirofilaria immitis) infection in dogs in northern Italy. Vet Parasitol. 2000;91(3-4):251-258.

12. Cringoli G, Rinaldi L, Veneziano V, Capelli G. A prevalence survey and risk analysis of filariosis in dogs from the Mt. Vesuvius area of southern Italy. Vet Parasitol. 2001;102(3):243-252.

13. Otranto D, Brianti E, Gaglio G, Dantas-Torres F, Azzaro S, Giannetto S. Human ocular infection with Dirofilaria repens (Railliet and Henry, 1911) in an area endemic for canine dirofilariasis. Am J Trop Med Hyg. 2011;84(6):1002-4. 
14. Benelli G, Romano D, Kavallieratos N, Conte G, Stefanini C, Mele M, et al. Multiple behavioural asymmetries impact male mating success in the khapra beetle, Trogoderma granarium. J Pest Sci 2017;90:901-909.

15. QGIS Development Team. QGIS geographic information system. Open Source Geospatial Foundation Project, Versão, 2015;2:7.

16. Maroli M, Rossi L, Baldelli R, Capelli G, Ferroglio E, Genchi C, et al. The northward spread of leishmaniasis in Italy: evidence from retrospective and ongoing studies on the canine reservoir and phlebotomine vectors. Trop Med Int Health. 2008;13(2):256-264.

17. Baldelli R, Piva S, Salvatore D, Parigi M, Melloni O, Tamba M, et al. Canine leishmaniasis surveillance in a northern Italy kennel. Vet Parasitol. 2011;179(1-3):57-61.

18. Morosetti G, Bongiorno G, Beran B, Scalone A, Moser J, Gramiccia M, Gradoni L, wt al. (2009). Risk assessment for canine leishmaniasis spreading in the north of Italy. Geospat Health. 2009;4(1):11527.

19. Signorini M, Cassini R, Drigo M, di Regalbono A, Pietrobelli M, Montarsi F, et al. Ecological niche model of Phlebotomus perniciosus, the main vector of canine leishmaniasis in north-eastern Italy. Geospatial health. 2014;193-201.

20. Genchi C, Solari Basano F, Marrone RV, Petruschke G. Canine and feline heartworm in Europe with special emphasis on Italy. In Proceedings of the heartworm symposium '98, Tampa, Florida, USA 1998;75-82.

21. Bourguinat C, Lee A, Lizundia R, Blagburn B, Liotta J, Kraus M, et al. Macrocyclic lactone resistance in Dirofilaria immitis: failure of heartworm preventives and investigation of genetic markers for resistance. Vet Parasitol. 2015;210(3-4):167-78.

22. Brandonisio O, Carelli G, Ceci L, Consenti B, Fasanella A, Puccini V. Canine leishmaniasis in the gargano promontory (Apulia, South Italy). Eur J Epidemiol.1992;8(2):273-276.

23. Vascellari M, Natale A, Schievenin E, Miatto A, Brino A, Frangipane Di Regalbono, et al. Descrizione di un nuovo focolaio di leishmaniosi canina nella Regione Veneto. Veterinaria. 2005;3:25-29.

24. Biglino A, Bolla C, Concialdi E, Trisciuoglio A, Romano A, Ferroglio E. Asymptomatic Leishmania infantum infection in an area of northwestern Italy (Piedmont region) where such infections are traditionally nonendemic. J Clin Microbiol. 2010;48(1):131-136.

25. Capelli G, Baldelli R, Ferroglio E, Genchi C, Gradoni L, Gramiccia M, et al. Monitoring of canine leishmaniasis in northern Italy: an update from a scientific network. Parassitologia. 2004;46(12):193-197.

26. Di Cesare A, Castagna G, Meloni S, Milillo P, Latrofa S, Otranto D, et al. Canine and feline infections by cardiopulmonary nematodes in central and southern Italy. Parasitol Res. 2011;109(1):87-96

27. Giangaspero A, Marangi M, Latrofa MS, Martinelli D, Traversa D, Otranto D et al. Evidences of increasing risk of dirofilarioses in southern Italy. Parasitol Res. 2013;112(3):1357-61.

28. Del Prete L, Maurelli MP, Pennacchio S, Bosco A, Musella V, Ciuca L, et al. Dirofilaria immitis and Angiostrongylus vasorum: the contemporaneous detection in kennels. BMC Vet Res. 2015;11(1):305. 
29. Sauda F, Malandrucco L, Macrì G, Scarpulla M, De Liberato C, Terracciano G, Fichi G, et al. Leishmania infantum, Dirofilaria and other endoparasite infections in kennel dogs in central Italy. Parasite. 2018;25:2.

30. Genchi M, Rinaldi L, Venco L, Cringoli G, Vismarra A, Kramer, L. Dirofilaria immitis and D. repens in dog and cat: a questionnaire study in Italy. Vet Parasitol. 2019;267:26-31.

31. Maresca C, Scoccia E, Barizzone F, Catalano A, Mancini S, Pagliacci T, et al. A survey on canine leishmaniasis and phlebotomine sand flies in central Italy. Res Vet Sci. 2009;87(1):36-8.

32. Vulpiani MP, lannetti L, Di Mattia T, Dalla Villa P. Leishmania infantum in a Central Italy dog shelter: retrospective study of serologic reactivity during a 4-year period in a confined dog population subjected to preventive and therapeutic treatment. Vet Parasitol. 2009;160(3-4):190-97.

\section{Table}

Table 1. Number and percentage ( $\mathrm{N} / \%)$ of serological tests positive for canine leishmaniosis and hearth worm disease in three main areas of Italy.

\begin{tabular}{llll}
\hline & North & Centre & South and Islands \\
& N $(\%)$ & N $(\%)$ & N $(\%)$ \\
\hline Leishmania infantum & $($ Tot=21,545) & $($ Tot=26,128) & $($ Tot=32,610) \\
& & & \\
\hline & $4,664(21.6)$ & $7,801(29.6)$ & $9,208(28.2)$ \\
\hline Dirofilaria immitis & $($ Tot=5,335) & $($ Tot $=3,119)$ & $($ Tot=1,866) \\
\hline & $151(2.8)$ & $243(7.5)$ & $93(4.9)$ \\
\hline
\end{tabular}

\section{Figures}




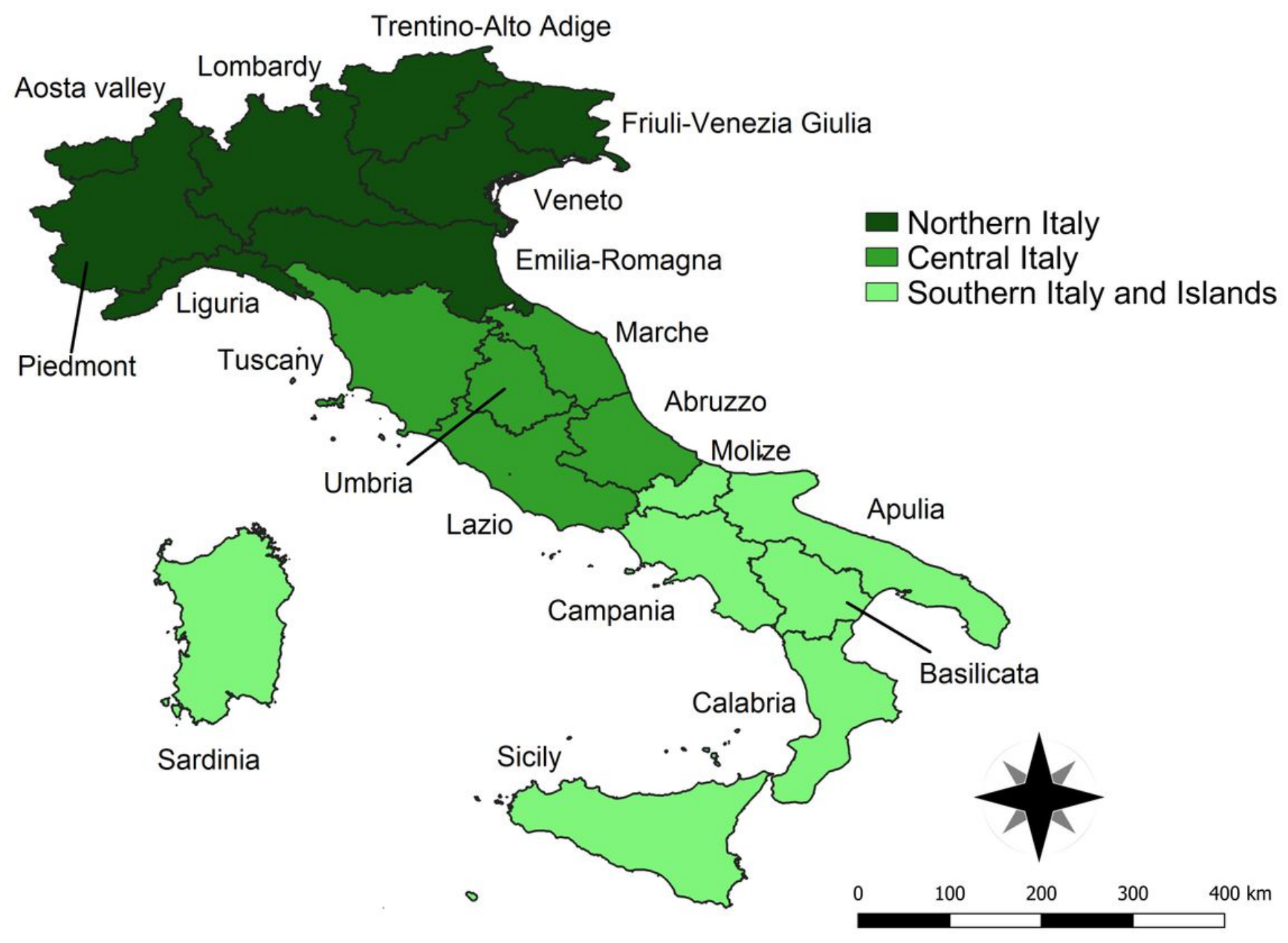

Figure 1

Italy. Three main areas with their respective administrative regions.

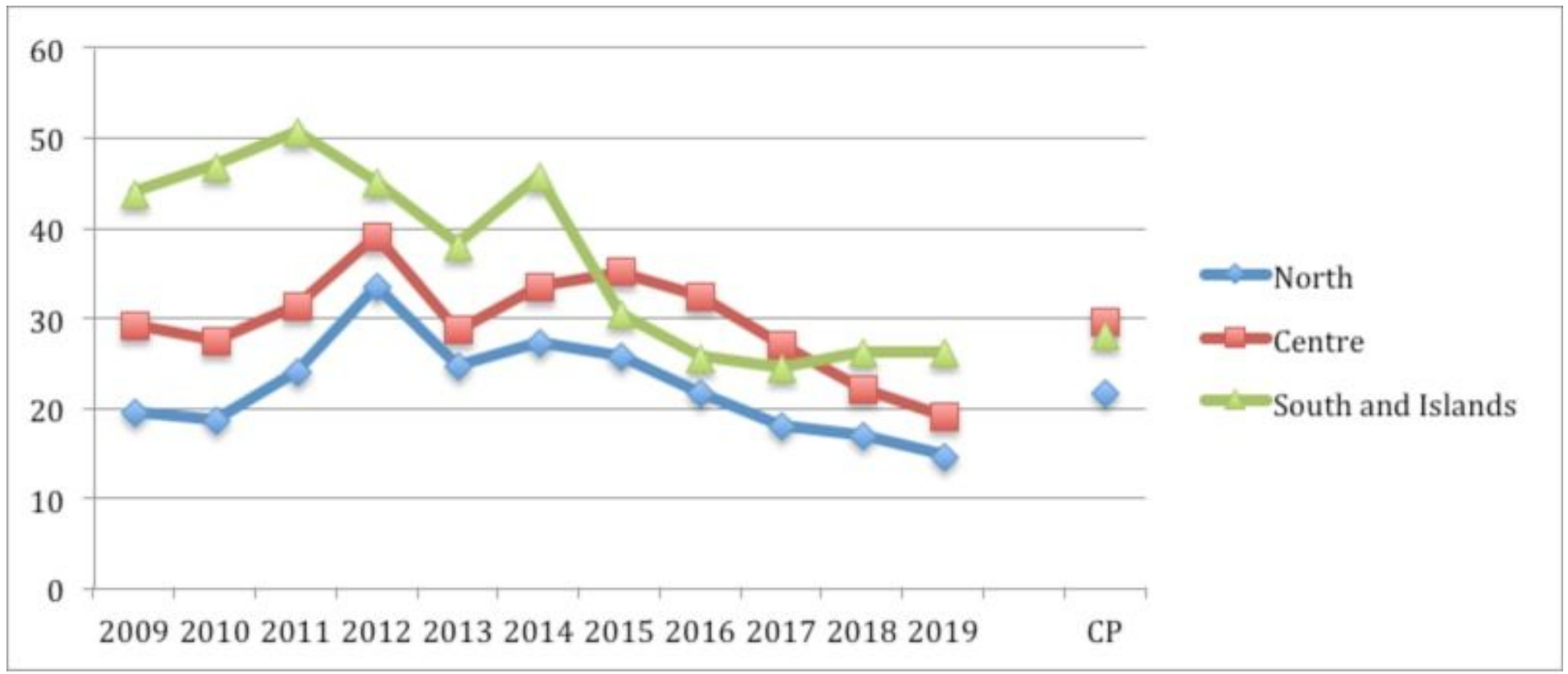


Figure 2

Pattern of mean annual seroprevalence of canine leishmaniosis in the three main regions of Italy. The cumulative prevalence (CP) is also reported.

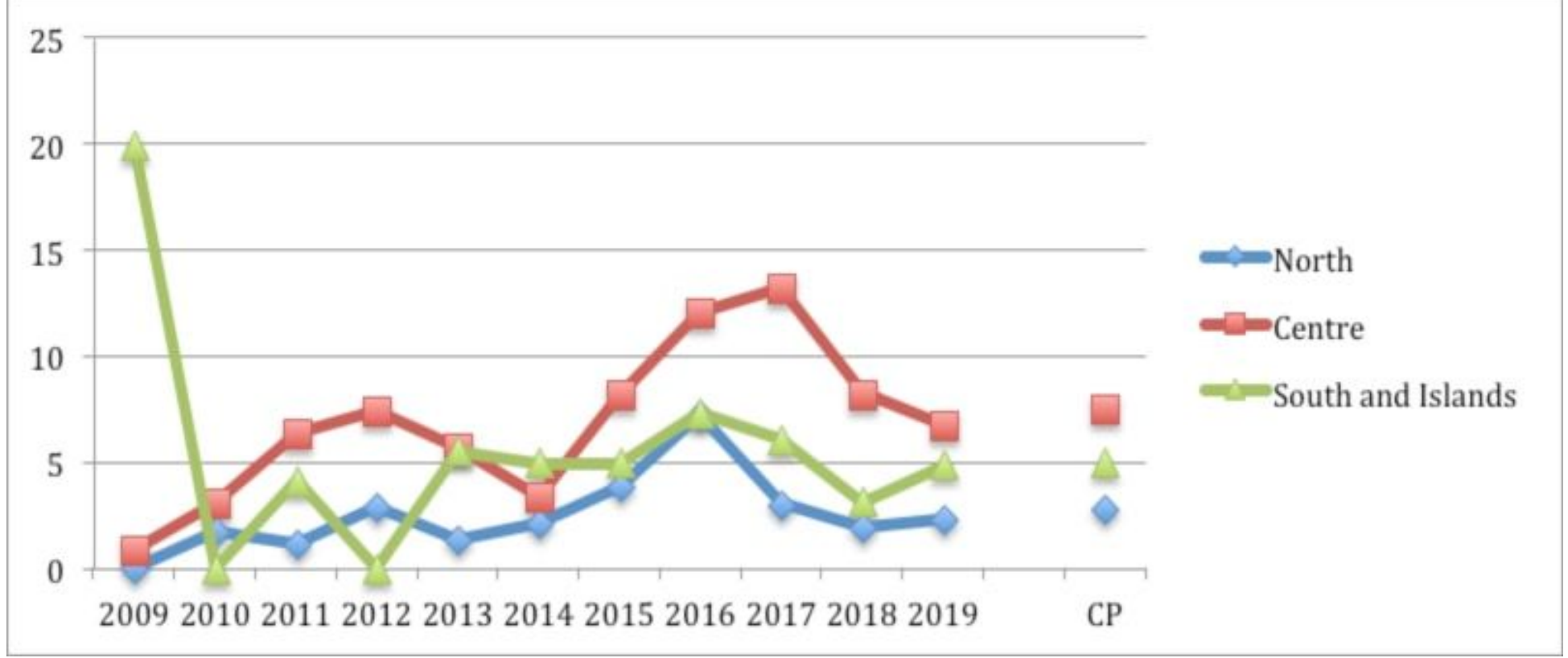

\section{Figure 3}

Pattern of mean annual seroprevalence for Dirofilaria immitis in the three main regions of Italy. The cumulative prevalence (CP) is also reported. 


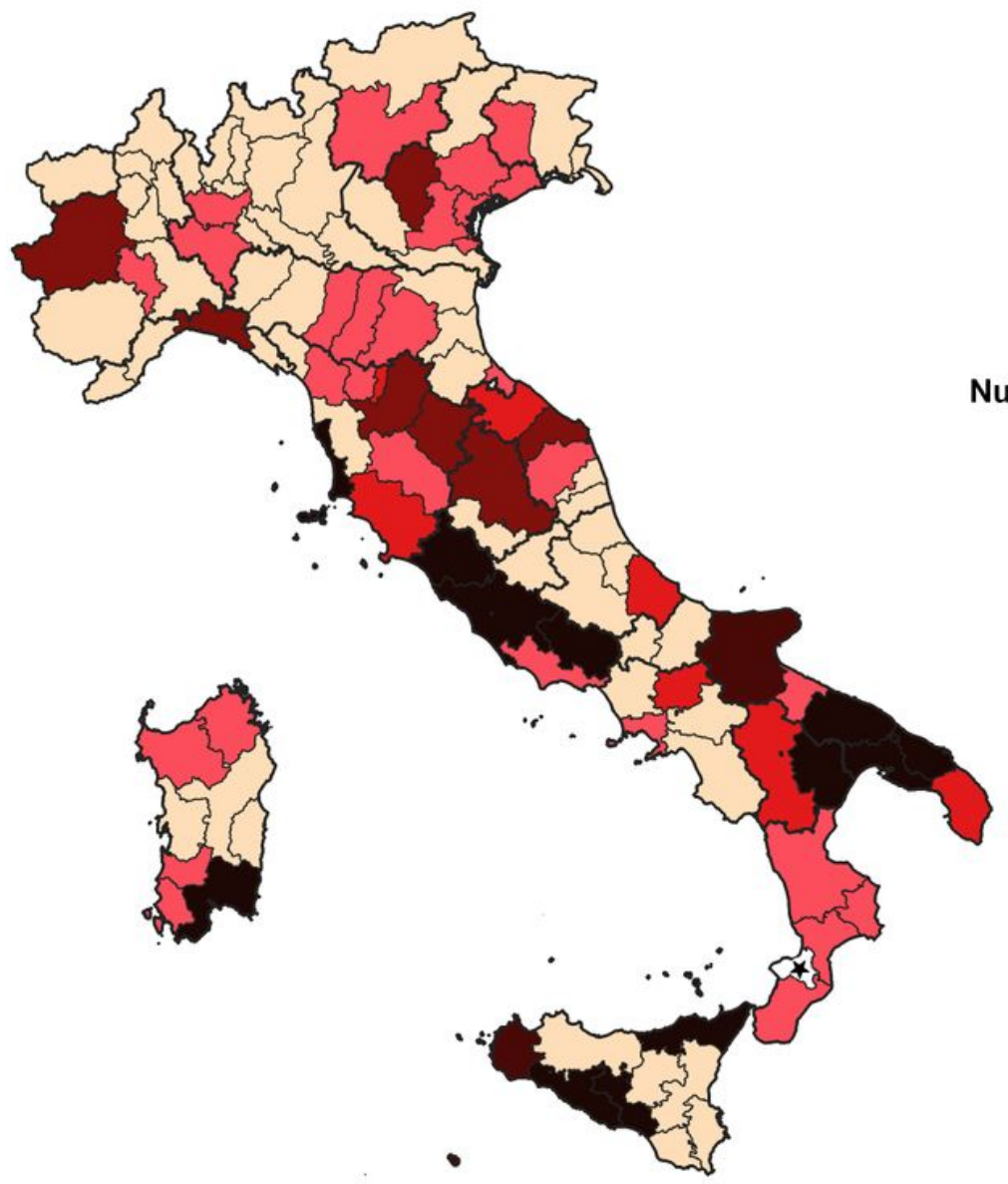

Number of positive samples (2009 - 2019)

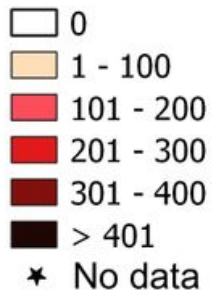

* No data
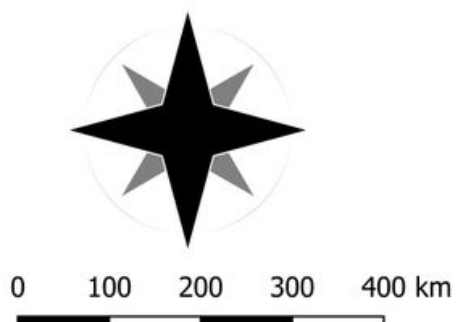

Figure 4

Distribution map with number of cases (2009 - 2019) per province of CanL in Italy. 


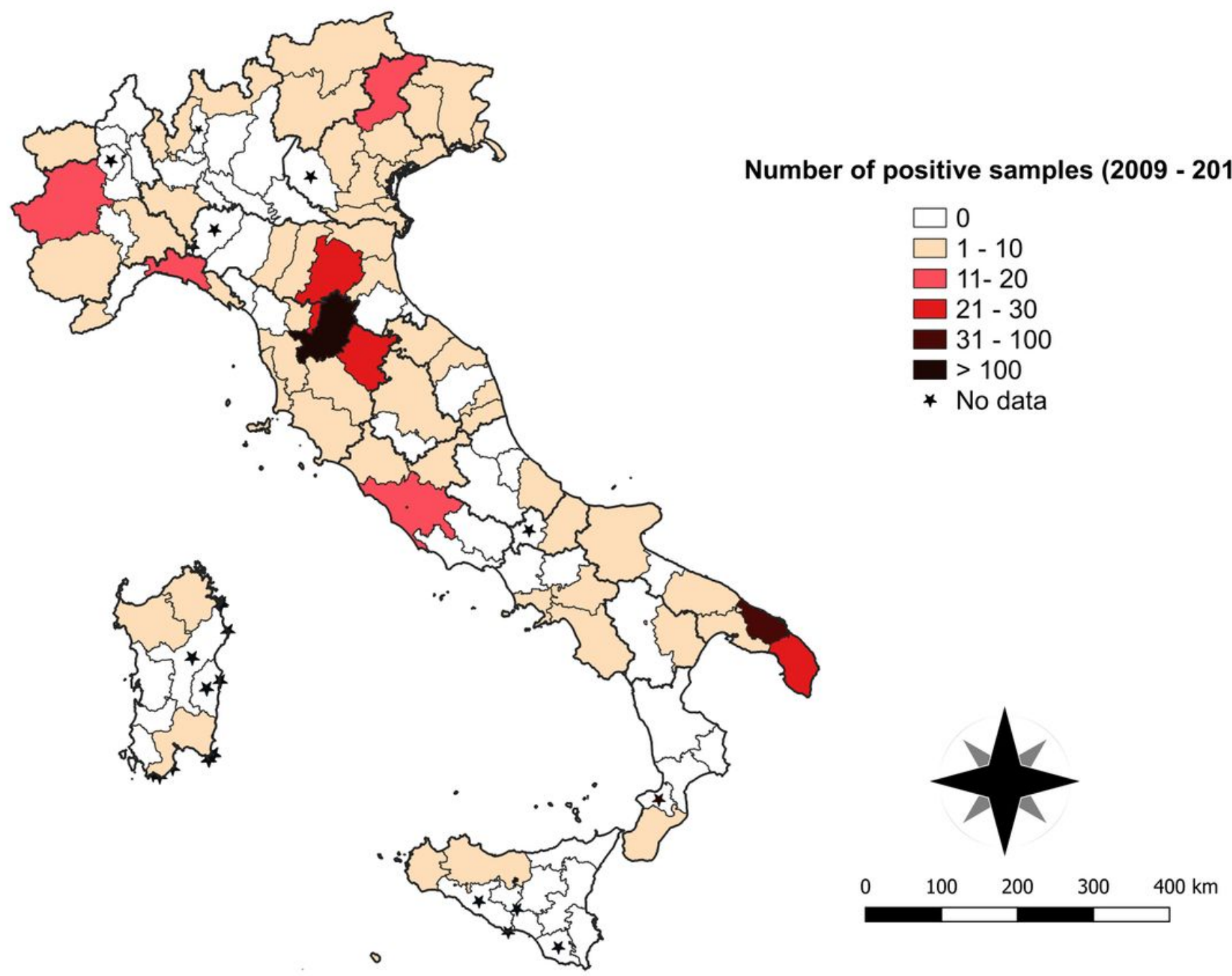

Figure 5

Distribution map with number of cases $(2009$ - 2019) per province of HW in Italy.

\section{Supplementary Files}

This is a list of supplementary files associated with this preprint. Click to download.

- Supplementarytable.docx 